\title{
Cerebrospinal Fluid Apolipoprotein E Levels in Delirium
}

\author{
Gideon A. Caplan ${ }^{a, b}$ JIan Taic Fazrul Mohd Hanizan ${ }^{c}$ \\ Catherine L. McVeigh ${ }^{a, b}$ Mark A. Hill ${ }^{c}$ Anne Poljak ${ }^{c-e}$ \\ a Department of Geriatric Medicine, Prince of Wales Hospital, Sydney, NSW, Australia; \\ b Prince of Wales Clinical School, University of New South Wales, Sydney, NSW, Australia; \\ 'School of Medical Sciences, University of New South Wales, Sydney, NSW, Australia; \\ dBioanalytical Mass Spectrometry Facility, University of New South Wales, Sydney, NSW, \\ Australia; ${ }^{\text {C} C e n t r e ~ f o r ~ H e a l t h y ~ B r a i n ~ A g e i n g, ~ U n i v e r s i t y ~ o f ~ N e w ~ S o u t h ~ W a l e s, ~ S y d n e y, ~ N S W, ~}$ \\ Australia
}

\section{Keywords}

Alzheimer disease $\cdot$ Delirium $\cdot$ Aged, 80 years and over $\cdot$ Spinal puncture $\cdot$ Biomarkers .

Apolipoprotein E4 $\cdot$ Third-party consent

\section{Abstract}

Background/Aims: Delirium and the apolipoprotein $\mathrm{E} \varepsilon 4$ allele are risk factors for late-onset Alzheimer disease (LOAD), but the connection is unclear. We looked for an association. Methods: Inpatients with delirium ( $n=18)$ were compared with LOAD outpatients $(n=19)$, assaying blood and cerebrospinal fluid (CSF) using multiplex ELISA. Results: The patients with delirium had a higher Confusion Assessment Method (CAM) score (5.6 \pm 1.2 vs. $0.0 \pm 0.0 ; p<$ 0.001 ) and Delirium Index (13.1 \pm 4.0 vs. $2.9 \pm 1.2 ; p=0.001)$ but a lower Mini-Mental State Examination (MMSE) score (14.3 \pm 6.8 vs. $20.8 \pm 4.6 ; p=0.003)$. There was a reduction in absolute CSF apolipoprotein E level during delirium (median [interquartile range]: $9.55 \mu \mathrm{g} / \mathrm{mL}$ [5.65-15.05] vs. $16.86 \mu \mathrm{g} / \mathrm{mL}$ [14.82-20.88]; $p=0.016$ ) but no differences in apolipoprotein $\mathrm{A} 1$, $\mathrm{B}, \mathrm{C} 3, \mathrm{H}$, and J. There were no differences in blood apolipoprotein levels, and no correlations between blood and CSF apolipoprotein levels. CSF apolipoprotein E correlated negatively with the CAM score $(r=-0.354 ; p=0.034)$ and Delirium Index $(r=-0.341 ; p=0.042)$ but not with the Acute Physiology and Chronic Health Evaluation (APACHE) index, or the MMSE or Informant Questionnaire on Cognitive Decline in the Elderly (IQCODE). Conclusion: Reduced CSF apolipoprotein E levels during delirium may be a mechanistic link between two important risk factors for LOAD. 


\section{Introduction}

Apolipoprotein E (ApoE) has an important role in regulating normal neuronal function through cholesterol transport and cell repair [1]. There are three ApoE isoforms - E2, E3, and $\mathrm{E} 4$ - derived from the apolipoprotein E gene (APOE) allele variants. The APOE $\varepsilon 4$ allele is the main genetic risk factor for late-onset Alzheimer disease (LOAD) [2]. Carriers have enhanced AD pathology and cognitive decline.

Apolipoproteins are unable to cross the blood-brain barrier; however, ApoE is expressed in the central nervous system (CNS) by astrocytes, microglia, and oligodendrocytes, where it transports phospholipids and cholesterol for neuronal membrane regeneration and remyelination. ApoE4 is more vulnerable to degradation than other isoforms, thereby limiting lipid mobilization for repair. The $\varepsilon 4$ allele is also associated with greater deposition of $A \beta$ in amyloid plaques and cerebral amyloid angiopathy, even in the absence of dementia [3].

Structural and functional brain changes associated with AD are detected earlier in $A P O E$ $\varepsilon 4$ carriers, before clinical symptoms develop $[1,4,5]$. APOE $\varepsilon 4$ contributes to AD pathogenesis by A $\beta$-independent mechanisms that involve synaptic plasticity, cholesterol homoeostasis, neurovascular functions, and neuroinflammation. ApoE $\varepsilon 4$ also interacts with modifiable risk factors for $\mathrm{AD}[5,6]$, and has been associated with other neurological disorders including Parkinson disease and with poor outcome after traumatic brain injury $[7,8]$. A recent meta-analysis found lower cerebrospinal fluid (CSF) ApoE levels in patients with AD in a subgroup analysis [9].

Delirium is a strong risk factor for dementia and a major acute driver of the progression of LOAD $[10,11]$ as measured by cognitive function, suggesting that crucial pathophysiological changes occur during delirium. One hypothesis characterizes delirium as an unmasking of an underlying, but as yet asymptomatic, cognitive decline [12]. Preventative efforts targeting patients at risk of delirium may slow the development of future dementia, and genetic variation could be a key determinant $[10,12,13]$.

Some studies support a link between the APOE \&4 allele and delirium [11, 14-16], while others do not [17-21], and meta-analyses [16,21] find a longer duration but not more frequent onset of delirium. Less information is available at the protein expression level as to whether CSF or plasma apolipoprotein expression is altered in delirium. Proteomics suggests that the levels of several apolipoproteins including ApoE are downregulated in CSF from subjects with delirium relative to pooled CSF from AD subjects [22]. Furthermore the APOE ع4 allele is associated with lower plasma levels of ApoE in normal older people [23].

Therefore, we sought to explore the relationship between ApoE levels in CSF and plasma in long-lasting delirium [24], defined as delirium which does not resolve after more than 5 days of treatment. Because ApoE cannot cross the blood-brain barrier [25], we hypothesized that changes to ApoE levels in the CSF would not be reflected in plasma ApoE concentrations. This is the first study to measure absolute levels of CSF ApoE in delirium.

\section{Subjects and Methods}

\section{Ethics Statement}

The guidelines of the World Medical Association Declaration of Helsinki - Ethical Principles for Medical Research Involving Human Subjects were adhered to in carrying out the work described in this paper. Participation in the study was discussed with each patient, where possible, and in all cases with their "person responsible" or substitute decision-maker, and informed consent was obtained. Ethics approval was obtained from the Human Research Ethics Committee of the South Eastern Sydney Health Area (Eastern Section) and the 
University of New South Wales Ethics Committee. The proposal was also reviewed by the NSW Guardianship Tribunal, which has to approve interventional studies involving participants who are unable to give consent, but not observational studies, and no objections were raised.

\section{Subjects and Clinical Evaluation}

Patients admitted to the Geriatric Medicine Unit at Prince of Wales Hospital were screened for delirium in the emergency department and on the geriatric medicine ward. Patients suffering from delirium (diagnosed by a geriatrician) which did not resolve after more than 5 days of treatment were considered for further investigation including lumbar puncture, and this was discussed with their "person responsible" or substitute decision-maker together with the patient, where possible.

Patients were suffering from a variety of active health problems including cardiac diseases, infections, electrolyte abnormalities, acute renal failure, falls, endocrine, haematological, respiratory, and surgical abdominal pathologies, fractures, neurological disorders, ethanol excess, and opiates. Each patient had at least two active problems, which could have been causally related to delirium, though often they were linked, for example, to a urinary tract infection and acute renal failure.

Our department minimizes the use of sedatives and antipsychotics, and the patients were taking the following psychoactive medications: narcotics (3 patients), selective serotonin reuptake inhibitors ( 2 patients), atypical antipsychotics ( 1 patient), sleeping tablets ( 1 patient), anticonvulsants (1 patient), acetylcholinesterase inhibitors (1 patient), and prednisone (1 patient). Most patients were suffering from mixed delirium with features of both hyper- and hypoalertness. When agreement with a more extensive diagnostic workup was given, entry into the study was offered, and written informed consent was obtained.

In consultation with their persons responsible, a group of outpatients with known AD, diagnosed according to the Diagnostic and Statistical Manual, 4th edition, and the National Institute of Neurological and Communicative Diseases and Stroke and the Alzheimer's Disease and Related Disorders Association criteria for probable AD, but no evidence of delirium, consented to act as controls.

A detailed baseline assessment included: the Confusion Assessment Method (CAM), which is a diagnostic instrument for delirium [26]; the Delirium Index to measure severity [27]; the Mini-Mental State Examination (MMSE) [28]; the Informant Questionnaire on Cognitive Decline in the Elderly (IQCODE) [29]; the Barthel Index, measuring basic activities of daily living function [30]; the modified Instrumental Activities of Daily Living (IADL) index [31]; the Acute Physiology and Chronic Health Evaluation (APACHE) II [32]; the Geriatric Depression Scale [33]; and the Charlson comorbidity index [34]. The IQCODE, Barthel, and IADL scores in the delirium group were obtained by asking carers about the patients' ability in the week or so prior to admission but before the deterioration associated with the admission had commenced. All data collection was done once, on the day of lumbar puncture.

\section{Plasma and CSF Sample Collection}

CSF samples were collected in the morning by lumbar puncture under X-ray guidance, using a local anaesthetic but no sedation or restraining devices. CSF (1-5 mL) was collected into polypropylene tubes, gently inverted $3-4$ times, and centrifuged $\left(2,000 \mathrm{~g}, 10 \mathrm{~min},+4^{\circ} \mathrm{C}\right)$ to remove cells and debris. The CSF was transferred to a clean polypropylene tube, and 50-, $100-$, and $250-\mu \mathrm{L}$ aliquots were dispensed into labelled cryovials and stored at $-80^{\circ} \mathrm{C}$ until required.

After lumbar puncture, blood was collected into an EDTA tube and gently inverted. Whole blood was stored at $+4{ }^{\circ} \mathrm{C}$ for no more than $4 \mathrm{~h}$, prior to separation of the plasma from cellular 
Table 1. Baseline characteristics

\begin{tabular}{lccc}
\hline & $\begin{array}{c}\text { Delirium } \\
(n=17)\end{array}$ & $\begin{array}{c}\text { Alzheimer disease } \\
(n=19)\end{array}$ & $p$ value \\
\hline Age, years & $83.8 \pm 5.8$ & $83.3 \pm 5.6$ & 0.853 \\
Sex (M:F) & $9: 8$ & $9: 10$ & 0.650 \\
History of dementia & 8 & 19 & 0.842 \\
Barthel Index & $18.3 \pm 2.7$ & $18.0 \pm 4.2$ & 0.915 \\
IADL index & $7.9 \pm 3.4$ & $7.8 \pm 3.2$ & 0.943 \\
Charlson comorbidity index & $5.8 \pm 3.0$ & $5.8 \pm 1.7$ & 0.075 \\
APACHE index & $39.8 \pm 11.5$ & $33.3 \pm 7.9$ & 0.800 \\
IQCODE score & $3.8 \pm 1.2$ & $3.7 \pm 0.5$ & $<\mathbf{0 . 0 0 1}$ \\
Confusion assessment method score & $\mathbf{5 . 6 \pm 1 . 2}$ & $\mathbf{0 . 0}$ & $\mathbf{< 0 . 0 0 0 1}$ \\
Delirium Index & $\mathbf{1 3 . 1 \pm \mathbf { 4 . 0 }}$ & $\mathbf{2 . 9 \pm \mathbf { 1 . 2 }}$ & 0.721 \\
Geriatric depression scale score & $6.4 \pm 5.1$ & $5.9 \pm 2.9$ & $\mathbf{0 . 0 0 3}$ \\
Mini-Mental State Examination score & $\mathbf{1 4 . 3 \pm 6 . 8}$ & $\mathbf{2 0 . 8 \pm 4 . 6}$ & \\
\hline
\end{tabular}

Values are presented as mean \pm SD or $n$. Bold type denotes significance. APACHE, Acute Physiology and Chronic Health Evaluation; IQCODE, Informant Questionnaire on Cognitive Decline in the Elderly.

components by centrifugation (10-20 min, 1,100-1,300 $\mathrm{g}$, ambient temperature). The plasma was transferred to a clean polypropylene tube, and 100 - and $1,000-\mu \mathrm{L}$ aliquots were dispensed into labelled cryovials and stored at $-80^{\circ} \mathrm{C}$ until used for the assay.

\section{Multiplex Fluorescence-Based ELISA of Apolipoproteins}

A commercially available multiplex kit was used for CSF and plasma apolipoprotein ELISA (WideScreen BeadPlex Human CVD Panel 1, catalogue No. BPHCVD01-7; Merck Millipore, Sydney, NSW, Australia). This kit is suitable for the assay of 7 apolipoproteins (ApoA1, ApoA2, ApoB, ApoC3, ApoE, ApoH, and ApoJ) and the plates were read on a Bio-Rad Bio-Plex analyser. The CSF was diluted by a factor of 1:200 and the plasma was diluted to 1:2,500; all samples were assayed in duplicate. The manufacturer's instructions were used, and we followed our previously published procedure [22,23]. The scientist conducting the assay was blinded to the delirium status of the patients.

\section{Statistical Analysis}

SPSS (version 20; SPSS Inc., Chicago, IL, USA) was used for statistical analyses. Normality of the distribution was checked by assessing kurtosis and skewness. All the CSF and blood apolipoprotein levels were normally distributed, except for CSF ApoE. Normally distributed continuous variables were compared using independent-samples $t$ tests. CSF ApoE was assessed using the independent-samples Kruskal-Wallis test. Bivariate Pearson correlation coefficients were used to compare continuous variables. All statistical tests were two-tailed, and any results with a $p$ value $\leq 0.05$ were considered statistically significant.

\section{Results}

The baseline subject group characteristics (Table 1) showed that the two groups were well matched for age, sex, and measures of ADL (the Barthel and IADL indices) as well as the Charlson comorbidity index. As expected, the delirium group had higher scores on measures of delirium, the CAM (delirium vs. AD group, mean \pm SD: $5.6 \pm 1.2$ vs. $0.0 \pm 0.0 ; p<0.001$ ) and 
Table 2. CSF apolipoprotein levels

\begin{tabular}{lccc}
\hline & Delirium $(n=17)$ & Alzheimer disease $(n=19)$ & $p$ value \\
\hline ApoA1, $\mu \mathrm{g} / \mathrm{mL}$ & $20.38 \pm 11.00$ & $24.65 \pm 8.48$ & 0.206 \\
ApoB, $\mu \mathrm{g} / \mathrm{mL}$ & $2.46 \pm 2.42$ & $1.96 \pm 1.20$ & 0.477 \\
ApoC3, $\mu \mathrm{g} / \mathrm{mL}$ & $0.13 \pm 0.90$ & $0.10 \pm 0.62$ & 0.337 \\
ApoE, $\mu \mathrm{g} / \mathrm{mL}$ & $\mathbf{1 1 . 2 4} \pm \mathbf{5 . 7 6}$ & $\mathbf{1 8 . 1 7} \pm \mathbf{1 0 . 4 9}$ & $\mathbf{0 . 0 2 2}$ \\
ApoH, $\mu \mathrm{g} / \mathrm{mL}$ & $1.72 \pm 0.89$ & $1.83 \pm 0.57$ & 0.688 \\
ApoJ, $\mu \mathrm{g} / \mathrm{mL}$ & $17.60 \pm 12.43$ & $25.64 \pm 13.76$ & 0.079 \\
AроB/ApoA1 & $0.11 \pm 0.09$ & $0.08 \pm 0.06$ & 0.408 \\
\hline
\end{tabular}

Values are presented as mean \pm SD. Bold type denotes significance. Apo, apolipoprotein.

Table 3. Plasma apolipoprotein levels

\begin{tabular}{lccc}
\hline & Delirium $(n=17)$ & Alzheimer disease $(n=19)$ & $p$ value \\
\hline ApoA1, $\mu \mathrm{g} / \mathrm{mL}$ & $1,270 \pm 360$ & $1,080 \pm 910$ & 0.718 \\
ApoA2, $\mu \mathrm{g} / \mathrm{mL}$ & $222.74 \pm 78.92$ & $260.73 \pm 96.62$ & 0.272 \\
ApoB, $\mu \mathrm{g} / \mathrm{mL}$ & $758.29 \pm 239.10$ & $679.98 \pm 213.52$ & 0.338 \\
ApoC3, $\mu \mathrm{g} / \mathrm{mL}$ & $20.82 \pm 5.25$ & $14.29 \pm 7.49$ & 0.153 \\
ApoE, $\mu \mathrm{g} / \mathrm{mL}$ & $32.35 \pm 32.52$ & $29.44 \pm 32.89$ & 0.818 \\
ApoH, $\mu \mathrm{g} / \mathrm{mL}$ & $153.97 \pm 55.73$ & $135.47 \pm 47.36$ & 0.327 \\
ApoJ, $\mu \mathrm{g} / \mathrm{mL}$ & $70.96 \pm 37.80$ & $72.34 \pm 34.72$ & 0.917 \\
ApoB/ApoA1 & $583.81 \pm 201.97$ & $497.25 \pm 420.23$ & 0.708 \\
\hline
\end{tabular}

Values are presented as mean \pm SD. Apo, apolipoprotein.

the Delirium Index $(13.1 \pm 4.0$ vs. $2.9 \pm 1.2 ; p<0.001)$. They also had a lower score on the MMSE $(14.3 \pm 6.8$ vs. $20.8 \pm 4.6 ; p=0.003)$, although there was no difference in IQCODE scores, and they showed a trend towards higher scores on the APACHE $(39.8 \pm 11.5$ vs. $33.3 \pm 7.9 ; p=0.075$ ).

On multiplex ELISA testing there was a significant reduction in absolute CSF ApoE levels in the subjects with delirium compared to those with $\mathrm{AD}$ (median [interquartile range]: 9.55 $\mu \mathrm{g} / \mathrm{mL}$ [5.65-15.05] vs. $16.86 \mu \mathrm{g} / \mathrm{mL}$ [14.82-20.88]; $p=0.016)$. There were no statistically significant differences in the levels of the other apolipoproteins assayed, though trends were seen for lower mean levels of the apolipoproteins A1, H, and J and a higher apolipoprotein B level with delirium than with AD. For ApoA2 the CSF results were beyond the range of the standard curve (Table 2).

There were no statistically significant differences in the blood levels of any of the apolipoproteins between the two groups (Table 3). There were also no significant correlations between the levels of any of the individual apolipoproteins in CSF and those in blood.

CSF ApoE levels correlated negatively with the clinical measures of delirium (Fig. 1) - i.e., the CAM ( $r=-0.354 ; p=0.034)$ and the Delirium Index ( $r=-0.341 ; p=0.042)$ - but not with the measure of acute illness, the APACHE index, or the measures of cognitive function or dementia (i.e., the MMSE or IQCODE). Additionally, when we dichotomized the IQCODE score into $<3.44$ or $\geq 3.44$, there was no difference in CSF ApoE levels $(16.6 \pm 12.3$ vs. $13.9 \pm 6.9$; $p=0.406$ ). 
Fig. 1. Severity of delirium versus cerebrospinal fluid (CSF) apolipoprotein E level. Pearson correlation coefficient $=-0.341 ; p=$ 0.042 .

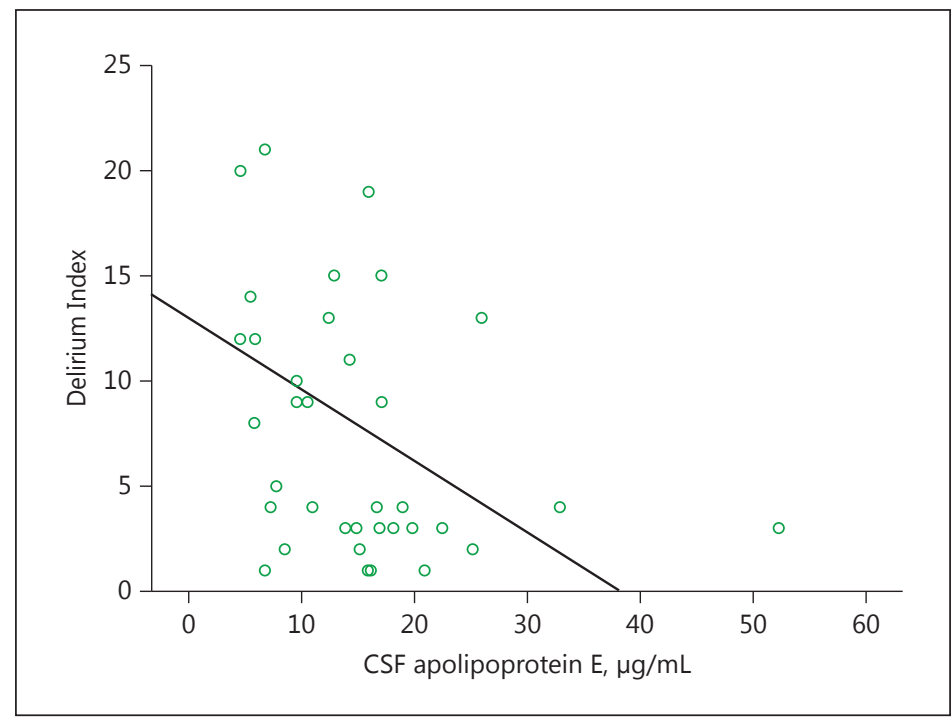

\section{Discussion}

This is the first study discovering lower levels of ApoE in CSF during delirium. This may be due to either increased turnover or reduced synthesis. APOE $\varepsilon 4$ is the main genetic risk factor for LOAD [1, 2], and lower levels of ApoE are observed in the plasma of APOE \&4 allele carriers, and more so among homozygous than among heterozygous carriers in a normal population [23]. ApoE levels have also been found to be lower in AD [35], suggesting an association of lower ApoE levels with neurodegenerative diseases. A lower CSF ApoE level in delirium may also reflect increased neuronal damage [3]. However, a meta-analysis of CSF ApoE levels in patients with $\mathrm{AD}$ overall found no association, unless the authors restricted the analysis to larger studies [36]. They found no evidence of lower CSF ApoE levels with greater severity of AD. Thus, although our delirium group had lower MMSE scores - likely due to the cognitive effects of delirium given that their IQCODE scores were not lower, but possibly due to more severe underlying dementia - there is no reason to ascribe the lower ApoE levels to more severe dementia.

The pathophysiology and mechanisms involved in the development of delirium are complex and the involvement of ApoE is no exception. This multifunctional protein has roles in modulation of inflammation, complement inactivation, $A \beta$ binding, and modulation of neuronal plasticity and apoptosis.

The association of $A P O E$ with LOAD is well documented and multifaceted. Ischaemic brain insults and hypertension-related white matter lesions (known contributors to severity of dementia in $\mathrm{AD}$ ) tend to be more pronounced in $A P O E \varepsilon 4$-positive patients. The enhanced formation of C-terminal-truncated fragments characteristic of the E4 isoform stimulates tau hyperphosphorylation and formation of neurofibrillary tangles [4].

However, the main role of ApoE in the CNS is transportation of cholesterol to neurons and cell wall repair, implicating neuronal damage in the pathophysiological contribution of ApoE to AD. The APOE \&4 allele enhances synaptic loss around plaques [35], and ApoE $\varepsilon 4$ facilitates the association of oligomeric $A \beta$ with synapses, where it is directly toxic. Synapse loss is a strong correlate of cognitive decline in $\mathrm{AD}$ [37], and an imaging study suggested synaptic dysfunction to occur decades before AD onset in $A P O E \varepsilon 4$ carriers [38]. 
ApoE also influences the interaction between modifiable risk factors (external to the CNS) in LOAD, such as glucose/insulin homeostasis, but also between non-modifiable risk factors such as sex hormones, hyperlipidaemia, nutritional factors, physical exercise, and traumatic brain injury, in the determination of $\mathrm{AD}$ risk and the response to intervention. Among both subjects with mild cognitive impairment and cognitively normal older females, but not among males, $A P O E \varepsilon 4$ carriers show a more rapid decline in cognitive function than do non-carriers. The $A P O E \varepsilon 4$ allele has also been linked with decreased cerebral glucose metabolism in normal people on FDG-PET $\left({ }^{18} \mathrm{~F}\right.$-fluorodeoxyglucose positron emission tomography) [5] and with higher rates of conversion into dementia in people with AD neuropathology [6].

The $A P O E \varepsilon 4$ allele is a well-established risk factor for LOAD [1-5]; while the relationship with delirium is less studied, the evidence is against a causative relationship. Thus, it is likely that its effects play out through altered expression levels or turnover of apolipoproteins, particularly in CSF. This is likely to be the functionally more important measure than genotype per se.

Perhaps this is because not only patients with AD develop delirium more readily. Any dementing process is a risk factor for delirium, and thus there should not be a presumption that the $A P O E \varepsilon 4$ allele is a risk factor for delirium in all people just because it is one for AD. People with Lewy body dementia, for example, are prone to delirium, perhaps thereby diluting the effect of $A P O E \varepsilon 4$ on delirium. Other, perhaps currently unrecognized, factors may also mitigate the relationship between $A P O E \varepsilon 4$ and delirium.

A number of pathophysiological mechanisms have been postulated to explain the relationship between ApoE and delirium [14], including the following:

1. Modulation of the inflammatory response of the brain and modification of glial activation

2. In already damaged brains, the presence of the $A P O E \varepsilon 4$ allele accelerates the promotion of $A \beta$ aggregation and fibril formation, leading to non-recovery from delirium and to dementia progression

3. The free radicals hypothesis

4. Blockage of nicotinic acetylcholine receptors, causing the anticholinergic effect which is assumed in delirium

Additionally, suppression of the cerebral glucose metabolism on FDG-PET scans in asymptomatic APOE \&4 carriers may constitute another pathophysiological mechanism [24]. All these theories posit a CNS involvement of ApoE in delirium. Therefore, our finding a link between CSF ApoE levels and delirium is likely to be a more accurate representation of altered signalling processes and brain pathophysiological changes. This is the major strength of our study.

The fact that there were no significant differences in apolipoprotein blood levels between the two groups and no correlations between the levels of the individual apolipoproteins in CSF and those in blood suggests that peripheral ApoE levels cannot measure brain dysfunction in delirium compared to dementia.

In our study, not only was the CSF ApoE level lower in delirium but it also negatively correlated with the severity of the delirium, supporting a possible pathophysiological role. Given that the decrease in cognitive and ADL function in delirium is worse with greater delirium severity (as well as likely arising from damage to the CNS) and the role of ApoE in neuronal maintenance and repair, the downregulation of ApoE in the CSF of delirium patients represents a novel potential mechanism linking neuronal damage and delirium.

The patients with delirium in our study were ipso facto acutely unwell, since this is part of the definition of delirium. It is possible that the change in ApoE levels may have been due to the underlying illness rather than due to the delirium. However, since the underlying illness causes the delirium, this may be more of a philosophical distinction. Additionally, because this 
was a group of acute geriatric medicine patients with a mixture of diagnoses, it is unlikely that one particular illness the group had in common apart from delirium was responsible.

The delirium symptoms of these patients were treated in the usual way, primarily by treating the underlying illnesses and with supportive measures. We earnestly try to minimize the use of sedatives, and the extent of their use is outlined in the Subjects and Methods.

Dementia is the most important risk factor for delirium. The patients in the delirium group actually had a score on the IQCODE similar to that of the group with dementia. This may indicate that they all had a pre-existing dementia, and that therefore the groups were well matched. The group with delirium had a significantly lower score on the MMSE, which could be interpreted as meaning that they had worse dementia, and that this may have accounted for the changes we found. However, their similar scores on the IQCODE make this unlikely, and we know that delirium also worsens cognitive function and is therefore sufficient to account for the worse MMSE scores. It is quite likely that the current episode of prolonged delirium influenced carers' responses to the IQCODE, even though we asked them to think about the patient's cognitive function 2 weeks before admission, because of the phenomenon of recency bias, where more recent events and observations have a disproportionate effect on people's opinions.

Our small study size is a limitation, as it reduces its statistical power. The trend toward lower levels of other apolipoproteins in delirium may have been significant in a larger group. It would also have been useful to have (1) a normal control comparison group, (2) a validation cohort from another centre, and (3) APOE genotype information for comparison with ApoE levels. These points could be addressed in future studies. Lumbar puncture in patients with delirium can be a difficult task, but even with small numbers we have shown an intriguing result which warrants further exploration.

\section{References}

1 Benarroch EE: Brain cholesterol metabolism and neurologic disease. Neurology 2008;71:1368-1373.

2 Ward A, Crean S, Mercaldi CJ, et al: Prevalence of apolipoprotein E4 genotype and homozygotes (APOE e4/4) among patients diagnosed with Alzheimer's disease: a systematic review and meta-analysis. Neuroepidemiology 2012;38:1-17.

-3 Polvikoski T, Sulkava R, Haltia M, et al: Apolipoprotein E, dementia, and cortical deposition of $\beta$-amyloid protein. N Engl J Med 1995;333:1242-1247.

4 Bennett DA, Wilson RS, Schneider JA, et al: Apolipoprotein E $\varepsilon 4$ allele, AD pathology, and the clinical expression of Alzheimer's disease. Neurology 2003;60:246-252.

5 Jagust WJ, Landau SM: Apolipoprotein E, not fibrillar $\beta$-amyloid, reduces cerebral glucose metabolism in normal aging. J Neurosci 2012;32:18227-18233.

-6 Monsell SE, Mock C, Roe CM, et al: Comparison of symptomatic and asymptomatic persons with Alzheimer disease neuropathology. Neurology 2013;80:2121-2129.

7 Zhou W, Xu D, Peng X, et al: Meta-analysis of APOE4 allele and outcome after traumatic brain injury. J Neurotrauma 2008;25:279-290.

8 Li YJ, Hauser MA, Scott WK, et al: Apolipoprotein E controls the risk and age at onset of Parkinson disease. Neurology 2004;62:2005-2009.

-9 Talwar P, Sinha J, Grover S, et al: Meta-analysis of apolipoprotein E levels in the cerebrospinal fluid of patients with Alzheimer's disease. J Neurol Sci 2016;360:179-187.

10 Davis DH, Muniz Terrera G, Keage H, et al: Delirium is a strong risk factor for dementia in the oldest-old: a population-based cohort study. Brain 2012;135(pt 9):2809-2816.

11 Ely EW, Girard TD, Shintani AK, et al: Apolipoprotein E4 polymorphism as a genetic predisposition to delirium in critically ill patients. Crit Care Med 2007;35:112-117.

12 Nadelson MR, Sanders RD, Avidan MS: Perioperative cognitive trajectory in adults. Br J Anaesth 2014;112:440451.

13 van Munster BC, Zwinderman AH, de Rooij SE: Genetic variations in the interleukin- 6 and interleukin-8 genes and the interleukin-6 receptor gene in delirium. Rejuvenation Res 2011;14:425-428.

14 Adamis D, Treloar A, Martin FC, et al: APOE and cytokines as biological markers for recovery of prevalent delirium in elderly medical inpatients. Int J Geriatr Psychiatry 2007;22:688-694. 
15 Leung JM, Sands LP, Wang Y, et al: Apolipoprotein E e4 allele increases the risk of early postoperative delirium in older patients undergoing noncardiac surgery. Anesthesiology 2007;107:406-411.

16 van Munster BC, Korevaar JC, Zwinderman AH, et al: The association between delirium and the apolipoprotein E $\varepsilon 4$ allele: new study results and a meta-analysis. Am J Geriatr Psychiatry 2009;17:856-862.

17 Tagarakis GI, Tsolaki-Tagaraki F, Tsolaki M, et al: The role of apolipoprotein E in cognitive decline and delirium after bypass heart operations. Am J Alzheimers Dis Other Demen 2007;22:223-228.

18 Bryson GL, Wyand A, Wozny D, et al: A prospective cohort study evaluating associations among delirium, postoperative cognitive dysfunction, and apolipoprotein E genotype following open aortic repair. Can J Anaesth 2011;58:246-255.

19 Oldenbeuving AW, de Kort PL, Kappelle LJ, et al: Delirium in the acute phase after stroke and the role of the apolipoprotein E gene. Am J Geriatr Psychiatry 2013;21:935-937.

20 Abelha FJ, Fernandes V, Botelho M, et al: Apolipoprotein E e4 allele does not increase the risk of early postoperative delirium after major surgery. J Anesth 2012;26:412-421.

21 Adamis D, Meagher D, Williams J, et al: A systematic review and meta-analysis of the association between the apolipoprotein E genotype and delirium. Psychiatr Genet 2016;26:53-59.

22 Poljak A, Hill M, Hall RJ, et al: Quantitative proteomics of delirium cerebrospinal fluid. Transl Psychiatry 2014; 4:e477.

23 Song F, Poljak A, Crawford J, et al: Plasma apolipoprotein levels are associated with cognitive status and decline in a community cohort of older individuals. PLoS One 2012;7:e34078.

24 Caplan GA, Kvelde T, Lai C, et al: Cerebrospinal fluid in long-lasting delirium compared with Alzheimer's dementia. J Gerontol A Biol Sci Med Sci 2010;65:1130-1136.

25 Zlokovic BV, Martel CL, Mackic JB, et al: Brain uptake of circulating apolipoproteins J and E complexed to Alzheimer's amyloid beta. Biochem Biophys Res Commun 1994;205:1431-1437.

26 Inouye SK, Dyck CH, van Alessi CA, et al: Clarifying confusion: the confusion assessment method. A new method for detection of delirium. Ann Intern Med 1990;113:941-948.

27 McCusker J, Cole M, Bellavance F, et al: Reliability and validity of a new measure of severity of delirium. Int Psychogeriatr 1998; 10:421-433.

28 Folstein MF, Folstein SE, McHugh PR: "Mini-mental state." A practical method for grading the cognitive state of patients for the clinician. J Psychiatr Res 1975;12:189-198.

29 Jorm A: The Informant Questionnaire on Cognitive Decline in the Elderly (IQCODE): a review. Int Psychogeriatr 2004;16:275-293.

30 Mahoney FI, Barthel DW: Functional evaluation: the Barthel Index. Md State Med J 1965;14:61-65.

-31 Lawton MP, Moss M, Fulcomer M, et al: A research and service oriented multilevel assessment instrument. J Gerontol 1982;37:91-99.

-32 Knaus WA, Draper EA, Wagner DP, et al: APACHE II: a severity of disease classification system. Crit Care Med 1985;13:818-829.

-33 Yesavage JA, Brink TL, Rose TL, et al: Development and validation of a geriatric depression screening scale: a preliminary report. J Psychiatr Res 1982-1983;17:37-49.

34 Charlson M, Pompei P, Ales KL, et al: A new method of classifying prognostic comorbidity in longitudinal studies: development and validation. J Chronic Dis 1987;40:373-383.

-35 Koffie RM, Hashimoto T, Tai HC, et al: Apolipoprotein E4 effects in Alzheimer's disease are mediated by synaptotoxic oligomeric amyloid- $\beta$. Brain 2012;135(pt 7):2155-2168.

-36 Talwar P, Sinha J, Grover S, et al: Meta-analysis of apolipoprotein E levels in the cerebrospinal fluid of patients with Alzheimer's disease. J Neurol Sci 2016;360:179-187.

-37 DeKosky ST, Scheff SW: Synapse loss in frontal cortex biopsies in Alzheimer's disease: correlation with cognitive severity. Ann Neurol 1990;27:457-464.

38 Gupta VB, Laws SM, Villemagne VL, et al: Plasma apolipoprotein E and Alzheimer disease risk: the AIBL study of aging. Neurology 2011;76:1091-1098. 